ARTICLE

\title{
Altered anticipation and processing of aversive interoceptive experience among women remitted from bulimia nervosa
}

\author{
Laura A. Berner ${ }^{1}$, Alan N. Simmons ${ }^{1,2}$, Christina E. Wierenga ${ }^{1,2}$, Amanda Bischoff-Grethe ${ }^{1}$, Martin P. Paulus ${ }^{1,3}$, \\ Ursula F. Bailer ${ }^{1,4}$ and Walter H. Kaye ${ }^{1}$
}

Bulimia nervosa $(\mathrm{BN})$ is characterized by dysregulated intake of food, which may indicate homeostatic imbalance. Critically important for homeostatic regulation is interoception, or the sensing and processing of body-relevant information. A welldocumented link between avoidance of unpleasant body sensations and BN symptoms suggests that aversive interoceptive experiences may be particularly relevant to BN pathophysiology. This study examined whether individuals with a history of BN show aberrant neural processing of aversive interoceptive stimuli. Using a cued inspiratory breathing load paradigm, we compared women remitted from BN (RBN; $n=24$; to reduce the confounding effects of active bulimic symptoms) and control women (CW; $n=25$ ). During breathing load anticipation, the RBN group, relative to $C W$, showed increased activation in mid-insula, superior frontal gyrus, putamen, dorsal anterior cingulate, posterior cingulate, and amygdala. However, over the course of the aversive experience, neural activation in RBN relative to CW showed an aberrant decline in most of these regions. Exploratory analyses indicated that greater activation during breathing load anticipation was associated with past bulimic symptom severity and the duration of symptom remission. An exaggerated anticipatory response and an abnormally decreasing response during aversive homeostatic perturbations may promote hallmark bulimic behaviors-binge eating, dietary restriction, and purging. Our findings support a role for homeostatic instability in $\mathrm{BN}$, and these altered patterns of brain activation may serve as novel targets for pharmacological, neuromodulatory, and behavioral interventions.

Neuropsychopharmacology (2019) 44:1265-1273; https://doi.org/10.1038/s41386-019-0361-4

\section{INTRODUCTION}

Bulimia nervosa (BN) is characterized by recurrent binge eating, compensatory behaviors like self-induced vomiting or restricted eating [1], and high levels of affective lability [2]. Interoception, or the detection and integration of signals related to body state (e.g., hunger, pain, heartbeat, and respiration [3]), critically determines one's experience of the body, supports both physiological state and emotion regulation [4], and drives approach toward and avoidance of food to promote homeostatic equilibrium. As such, a recent model of $\mathrm{BN}$ informed by a systematic review posits that altered interoception plays a vital role in the disorder's pathophysiology [5]. In line with this hypothesis, women with current or past BN show altered pain thresholds [6, 7], sensitivity to gastric distention [8], and heartbeat detection accuracy ([9], but see [10]) relative to controls. However, prior studies of interoception in BN rely on self-report and may reflect disturbances in cognitively mediated, conscious interoceptive experience rather than in basic processes underlying interoception.

As interoception is mostly unconscious, neuroimaging is particularly useful for interoceptive assessment [11]. The difference between anticipated and experienced interoceptive stimulation is thought to be computed in the mid- to posterior insula, with afferents and efferents to anterior and posterior insula and the anterior cingulate, where an error signal is generated [12]. The amygdala and striatum are also involved in encoding the predicted and experienced valence and intensity of salient stimuli $[3,13-16]$. Projections from anterior and mid-cingulate and lateral prefrontal cortex (PFC) to anterior insula likely regulate cognitive reactions during interoceptive experiences [15, 17], and rostral anterior cingulate, lateral PFC, and anterior insula are engaged during return to homeostasis after interoceptive perturbations [18]. Structural neuroimaging data indicate increased volume and white matter connectivity of the insula in BN $[19,20]$. In addition, functional imaging results consistently suggest that neural activation during taste anticipation and receipt in the anterior, mid-, and posterior insula, ventral striatum, medial frontal gyrus, cingulate, and amygdala is altered in BN relative to controls [21-25]. This aberrant activation during both the anticipation and processing of taste stimulation has been postulated to promote or perpetuate BN symptoms.

However, prior neuroimaging investigations of sensation processing in $\mathrm{BN}$ are limited in three critical ways. First, all functional neuroimaging studies of interoception in BN to date have focused on taste. There are multiple definitions of interoception, only some of which include taste $[11,26]$, and no studies of BN have assessed the neural correlates of non-disorderspecific senses that are universally included in the definition of interoception (e.g., pain, heartbeat, and air hunger).

\footnotetext{
${ }^{1}$ Department of Psychiatry, University of California, San Diego, Chancellor Park, 4510 Executive Dr., Suite 330, San Diego, CA 92121-3021, USA; ${ }^{2}$ VA San Diego Healthcare System,

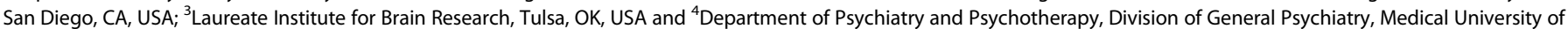
Vienna, Vienna, Austria

Correspondence: Walter H. Kaye (wkaye@ucsd.edu)
}

Received: 18 September 2018 Revised: 10 February 2019 Accepted: 12 February 2019

Published online: 6 March 2019 
Second, most studies have focused on pleasant experiences, but little is known about the neural processing of unpleasant interoceptive states in BN. Theoretical models posit that bulimic behaviors may represent efforts to distract from or otherwise avoid aversive cognition/affect and body sensations (e.g., fullness; [27]), and data support a strong association between avoidance of unpleasant internal experiences and increased bulimic symptomatology [28]. In addition, the tendency to act rashly when under distress is a major contributor to the development and maintenance of BN symptoms [29]. Taken together, these findings suggest that understanding how neural activation may be altered in BN during aversive, distressing internal experiences could inform a mechanistic understanding of the disorder.

Third, no studies have examined neural activation during anticipation and experience of aversive internal states in BN. Adaptive learning from body-related experience is thought to require the effective integration of neural signals for expectations with neural signals for the experience [12]. Recent theories posit that the disparity between expected and sensed aversive interoceptive states may promote a wide range of maladaptive behaviors intended to change the internal milieu to match anticipated states (e.g., binge eating, self-injury, and substance use) $[11,30]$. Therefore, examination of neural activation during both anticipation and processing of non-food specific, unpleasant interoceptive events is an important first step toward elucidating one of likely many dysfunctional aspects of interoception that could contribute to entrenched BN symptoms.

In the current study, we used a well-validated cued breathing load task to examine whether BN is associated with altered neural response before, during, and after aversive interoceptive stimulation. Unlike other powerful interoceptive probes (e.g., ballooninduced gastric distention), this task noninvasively creates extended, intermittent, unpleasant internal state changes. Prior reports have documented altered neural activation in other psychiatric populations using this task (Table S1). For example, adults with substance use disorders show reduced activation across all conditions of the task [31, 32], whereas we found that women remitted from AN (RAN) show a reduced anticipatory response and steeply increasing activation during the breathing load [33]. As in this prior investigation of RAN [33], the current study included only remitted participants to avoid confounding effects of active symptoms on neural function. Given that BN and substance use disorders may share neurobiological vulnerability factors [34] and both are characterized by impulsivity [35], we predicted that RBN, like adults with substance use disorders, would show reduced activation during all conditions of the task in the insula, lateral PFC, cingulate, and striatum [31, 32]. In light of amygdala contributions to aversive learning [36], we also predicted that RBN would show reduced amygdala activation across conditions. Secondary analyses tested the non-directional hypothesis that the time course of the blood oxygenation leveldependent (BOLD) response from anticipation to post-breathing load in RBN would differ from that of controls. Exploratory analyses examined associations of current and past clinical symptoms and self-report ratings with task-related activation.

\section{MATERIALS AND METHODS}

\section{Participants}

Twenty-five RBN and 26 healthy control women (CW) participated in this study. CW were recruited from San Diego, and RBN participants were recruited nationally. Masters-level or higher research staff administered standard structured interviews to confirm past eating disorder diagnosis and exclude current eating disorder diagnoses. RBN participants maintained more than $85 \%$ ideal body weight, regular menstrual cycles, and had no eating disorder behaviors for the 12 months before participation. Women who met diagnostic criteria for a current Diagnostic and Statistical Manual of Mental Disorders: Fourth Edition (DSM-IV) [37] Axis I diagnosis were excluded from both groups, and CW with a history of any eating disorder were excluded (see Supplement for further detail). Participants provided written, informed consent to participate in the study. The research was approved by the University of California, San Diego Human Research Protections Program.

\section{Procedure}

Aversive inspiratory breathing load paradigm. Within the first 10 days (early follicular phase) of their menstrual cycles, we scanned participants during an inspiratory breathing load fMRI paradigm (Fig. 1 and Supplement [31, 38-42]). Participants wore a nose clip while breathing through a hose that intermittently restricted breathing for cued 40-s periods. Participants completed a continuous performance task (CPT) to ensure attention to cues that predicted upcoming breathing loads (Fig. 1). Before scanning, participants were told that blue rectangles indicated no upcoming breathing load, and yellow rectangles signaled a one in four (25\%) chance of subsequent breathing load. Cardiac and respiratory data

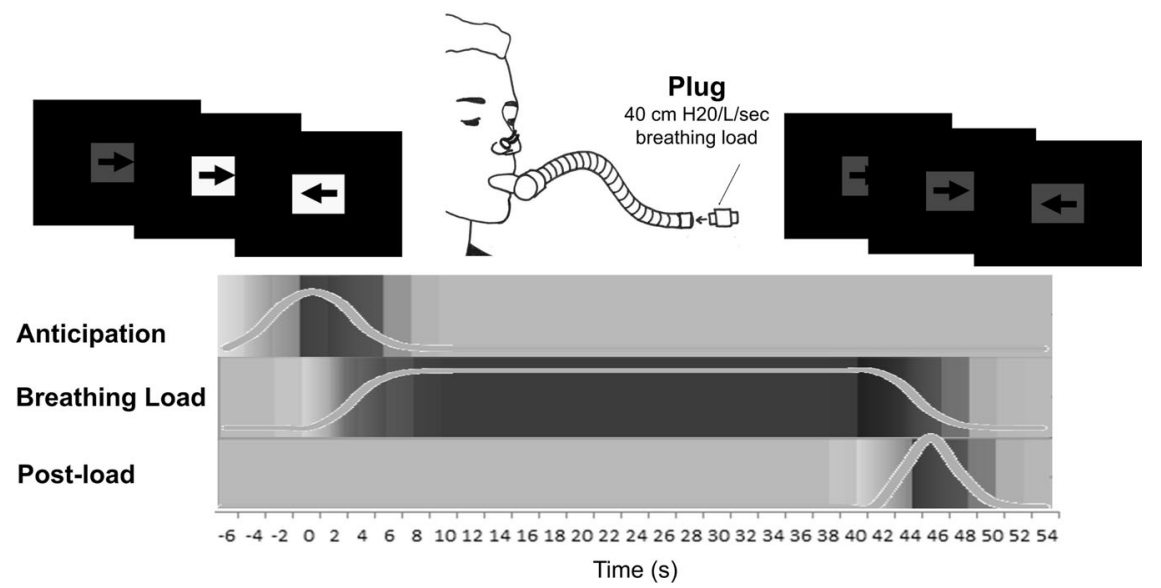

Fig. 1 Experimental paradigm. The inspiratory breathing load paradigm has been used to study aversive interoception in healthy and psychiatric populations [32, 38-42]. During fMRI scanning, participants wore a nose clip and were instructed to breathe through a hose that intermittently restricted breathing for $40-\mathrm{s}$ periods via $40 \mathrm{~cm} \mathrm{H} 20 / \mathrm{L} / \mathrm{sec}$ inspiratory loads. Colored rectangles (mean duration $6 \mathrm{~s}$ ) signaled the likelihood of an upcoming breathing load period (yellow $=25 \%$ chance of subsequent breathing load, blue $=0 \%$ chance). Overlaid on each rectangle, an arrow pointing left or right cued participants to press a left button or a right button, respectively. Accuracy and reaction time on this continuous performance task measured attention. The post-load period after all breathing load conditions lasted an average of $2 \mathrm{~s}$ 
were acquired using a fingertip pulse oximeter and a pneumatic belt around the upper abdomen, respectively.

Clinical measures. Participants completed the Eating Disorder Inventory-2 [43], Beck Depression Inventory (BDI-II; [44]), Temperament and Character Inventory (TCI) Harm Avoidance subscale (TCl$\mathrm{HA}$; [45]), and Spielberger State-Trait Anxiety Inventory (STAl; [46]). After first experiencing breathing load pre-scan and again immediately after scanning, participants rated the pleasantness, unpleasantness, and intensity of the experience using $10-\mathrm{cm}$ visual analogue scales (VAS). Additional post-scan VAS ratings of feelings of faintness, choking, abdominal distress, chest pain, and heart palpitations were also collected.

MRI statistical analyses

Imaging data (see Supplement for fMRI protocol detail) were analyzed with AFNI, FSL, and R statistical packages.

For primary, between-group analyses, three regressors of interest (anticipation, breathing load, and post-breathing load), and eight regressors of no interest (the "post-anticipation" condition when a yellow rectangle was not followed by breathing load, six motion regressors, and a baseline/linear drift regressor) were convolved with a modified hemodynamic response function using AFNI's 3dDeconvolve. The active baseline condition, when participants performed the CPT with a blue background, served as the baseline for within-subject imaging analyses [41]. Primary between-group $t$-tests (using AFNI's 3 dttest ++ ) within each condition examined whether groups differed in mean peak activation during breathing load anticipation, breathing load, and after breathing load termination.

Secondary analyses examined potential group differences in the time course of BOLD responses from anticipation to postbreathing load. Time-course data were modeled within each individual using AFNI's 3dDeconvolve TENT function. This approach modeled the hemodynamic response time-locked to the experimental design (anticipatory cue, 40-s aversive breathing load, post-breathing load). A Group $x$ Time linear mixed effects analysis (LME) conducted in AFNI's 3dLME [47] compared timecourse data from RBN and CW (see Supplement for additional detail).

As in our prior investigation of RAN [33], to limit multiple comparisons, we restricted primary and secondary fMRI analyses a priori to six bilateral regions (derived from the Harvard-Oxford atlas [48]) involved in the anticipation, processing, evaluation, and regulation of interoceptive state changes $[3,12-18,49]$ : (1) the insula in its entirety; (2) anterior/mid-cingulate (ACC); (3) posterior cingulate (PCC); (4) lateral PFC; (5) striatum, including the caudate, putamen, and ventral striatum; and (6) amygdala (Figure S1). Intrinsic smoothness was estimated using the spatial autocorrelation function (ACF) option in AFNI's 3dFWHMx. Results in each region were then small-volume corrected for multiple comparisons using Monte Carlo simulations via AFNI's 3dClustSim, including ACF estimates [50]. First-nearest neighbor clustering and a per-voxel threshold of $p<0.001$ (two-tailed) was set for each of the six search regions with a cluster-wise two-tailed alpha threshold of 0.05 . We also conducted an exploratory whole-brain Group (CW, RBN) x Condition (anticipation, breathing load, postbreathing load) LME analysis (see Supplement).

Clinical variables and potential confounds

Within the RBN group, exploratory negative binomial regressions conducted in $\mathrm{R}$ examined associations of percent signal change during breathing load anticipation, breathing load, and postbreathing load with bulimic symptom frequency (worst past binge eating frequency and self-induced vomiting frequency, measured in number of episodes per week). Huber robust regressions [51] examined associations of percent signal change with duration of BN remission. Sensitivity analyses (Huber robust regressions) examined the potential impact of lowest post-pubertal body mass index (BMI), VAS ratings, current depressive and anxiety symptoms, years of education, and CPT performance on our findings of between-group differences. Additional sensitivity analyses examined the potential confounding impact of past comorbidities (AN, major depression, and substance use disorders; see Supplement). As above, significant clusters were identified within each ROI using AFNI's 3dClustSim for small-volume correction (per voxel $p<0.001$ ). To control for family-wise error, within-group analyses were also Bonferroni-corrected for the number of clinical assessments and conditions tested. We computed the intersection of clusters identified in the primary task-related $t$-tests with those from regression analyses to assess for potential overlap. As both maps include only significant clusters, the resultant overlap also may be considered statistically significant [52].

\section{RESULTS}

Participants

One RBN and one CW were excluded from analyses because they did not complete both task runs. The final sample included 24 RBN (12 who also had a history of AN) and 25 CW (all included in a previous comparison with RAN [33]). Groups did not differ in age $(\mathrm{RBN}$ mean $=27.5 ; \mathrm{CW}$ mean $=25.2 ; p=0.464)$ or $\mathrm{BMI}(\mathrm{RBN}$ mean $=22.4 ; \mathrm{CW}$ mean $=22.0 ; p=0.372 ;$ Table S2). Past mood, substance, and alcohol use disorders were more common in the RBN group, and available data suggested that compared with CW, the RBN group reported higher, but non-clinically significant, levels of depressive symptoms, trait anxiety, and harm avoidance (Table S2).

Task performance, VAS ratings, and physiology

Across all task conditions, the RBN group responded more accurately than CW on the CPT $(p=0.046)$; however, both groups performed with high accuracy (RBN mean: 99.7\%; CW mean: 98.9\%), and groups did not differ on reaction time, nor was there a Group x Condition interaction on CPT performance measures (Table S3). RBN tended to rate post-scan feelings of faintness, choking, and heart palpitations more strongly than did CW, but these differences did not survive multiple comparisons correction, and groups did not differ on ratings of chest pain, abdominal distress, or the pleasantness, unpleasantness, or intensity of the breathing load experience (Table S3).

Groups did not differ on average (RBN: $0.5 \pm 0.4 \mathrm{~mm}$; CW: $0.6 \pm$ $0.4 \mathrm{~mm} ; p=0.310$ ) or maximum (RBN: $1.7 \pm 0.9 \mathrm{~mm}$; CW: $1.7 \pm$ $0.9 \mathrm{~mm} ; p=0.888$ ) motion during the task. Because of hardware malfunction, physiological data could be analyzed for only a subset of participants; however, available data provide some indication that groups did not differ in heart rate, breathing rate, or breathing rate variability (Supplement).

Within-condition analyses

During breathing load anticipation, RBN showed increased activation compared with $\mathrm{CW}$ in the bilateral mid- and left posterior insula, left superior frontal gyrus (SFG), dorsal ACC $(\mathrm{dACC}) /$ mid-cingulate, PCC, bilateral putamen, and right amygdala (Table 1 and Fig. 2). We did not detect group differences during the breathing load or post-load conditions within our regions of interest (see Figure S2 for effect size maps of non-statistically significant effects). Results of exploratory Group x Condition whole-brain, voxel-wise analyses are in Table S4.

Time course of activation across conditions

Group $\mathrm{x}$ time interactions were detected in left posterior insula, rostral and dACC, right middle frontal gyrus (MFG), bilateral PCC, and right amygdala (Table 2). CW activation in these areas gradually increased over the course of the task and peaked after 
Table 1. Between-group, within-condition differences in activation in a priori regions of interest

\begin{tabular}{|c|c|c|c|c|c|c|c|c|}
\hline \multirow[b]{2}{*}{ Hem } & \multirow[b]{2}{*}{ Region } & \multirow[b]{2}{*}{ BA } & \multirow[b]{2}{*}{ voxels } & \multicolumn{3}{|c|}{$\begin{array}{l}\text { Center of mass } \\
\text { MNI } \\
\text { coordinates }\end{array}$} & \multirow[b]{2}{*}{$t$} & \multirow[b]{2}{*}{$\begin{array}{l}\text { Cluster } \\
\text { Cohen's } d\end{array}$} \\
\hline & & & & $x$ & $y$ & $z$ & & \\
\hline \multicolumn{9}{|c|}{ Anticipation } \\
\hline $\mathrm{L}$ & Mid-insula & 13 & 23 & -34 & -4 & 1 & $3.87^{\mathrm{a}}$ & 1.13 \\
\hline $\mathrm{R}$ & Mid-insula & 13 & 17 & 35 & 0 & 5 & $3.78^{\mathrm{a}}$ & 1.10 \\
\hline $\mathrm{L}$ & Mid-insula & 13 & 12 & -39 & -5 & 11 & $3.78^{a}$ & 1.10 \\
\hline $\mathrm{L}$ & $\begin{array}{l}\text { Posterior } \\
\text { insula }\end{array}$ & 13 & 6 & -33 & -22 & 8 & $3.70^{a}$ & 1.08 \\
\hline $\mathrm{L}$ & $\begin{array}{l}\text { Superior } \\
\text { frontal gyrus }\end{array}$ & 6 & 19 & -30 & 13 & 53 & $3.75^{\mathrm{a}}$ & 1.09 \\
\hline $\mathrm{L}$ & Cingulate & 24 & 7 & -8 & -6 & 31 & $4.24^{\mathrm{a}}$ & 1.24 \\
\hline $\mathrm{R}$ & Cingulate & 24 & 7 & 6 & -7 & 42 & $3.64^{\mathrm{a}}$ & 1.06 \\
\hline $\mathrm{R}$ & Cingulate & 25 & 4 & 2 & 11 & -6 & $3.70^{\mathrm{a}}$ & 1.08 \\
\hline $\mathrm{R}$ & Cingulate & 24 & 4 & 2 & 2 & 42 & $3.77^{\mathrm{a}}$ & 1.10 \\
\hline $\mathrm{L}$ & $\begin{array}{l}\text { Posterior } \\
\text { cingulate }\end{array}$ & 31 & 8 & -12 & -33 & 39 & $3.68^{a}$ & 1.07 \\
\hline $\mathrm{R}$ & $\begin{array}{l}\text { Posterior } \\
\text { cingulate }\end{array}$ & 23 & 5 & 8 & -40 & 26 & $3.63^{\mathrm{a}}$ & 1.06 \\
\hline $\mathrm{L}$ & $\begin{array}{l}\text { Posterior } \\
\text { cingulate }\end{array}$ & 31 & 4 & -14 & -43 & 26 & 3.96 & 1.16 \\
\hline $\mathrm{R}$ & $\begin{array}{l}\text { Posterior } \\
\text { cingulate }\end{array}$ & 23 & 4 & 8 & -21 & 30 & 3.81 & 1.11 \\
\hline $\mathrm{L}$ & $\begin{array}{l}\text { Posterior } \\
\text { cingulate }\end{array}$ & 31 & 3 & -4 & -33 & 31 & 3.72 & 1.09 \\
\hline $\mathrm{L}$ & Putamen & & 10 & -30 & -4 & 5 & $3.87^{\mathrm{a}}$ & 1.13 \\
\hline $\mathrm{R}$ & Putamen & 13 & 5 & 30 & 3 & 7 & $3.69^{\mathrm{a}}$ & 1.08 \\
\hline $\mathrm{R}$ & Putamen & & 4 & 29 & -5 & 3 & $3.85^{\mathrm{a}}$ & 1.12 \\
\hline $\mathrm{R}$ & Putamen & & 4 & 29 & -10 & 7 & $3.60^{\mathrm{a}}$ & 1.05 \\
\hline$L$ & Putamen & & 3 & -25 & -8 & 9 & 3.76 & 1.10 \\
\hline $\mathrm{R}$ & Amygdala & & 2 & 20 & -9 & -18 & 4.44 & 1.29 \\
\hline
\end{tabular}

Positive $t$ statistics indicate that activation in the group of women remitted from bulimia nervosa is greater than that of controls. No statistically significant between-group differences were found during or after the breathing load. All results presented were small-volume corrected with Monte Carlo simulations (via Analysis of Functional Neurolmages 3dClustSim with ACF correction) to guard against false-positive results (per voxel $p<0.001$, cluster-wise $p<0.05$ ). Coordinates are presented in LPI format

Hem hemisphere, $L$ left, $R$ right, $B A$ Brodmann area

${ }^{a}$ Clusters that are statistically significant after more stringent small-volume correction (per voxel $p<0.001$, cluster-wise $p<0.01$ )

breathing load offset. In contrast, in all areas except the amygdala, RBN activation peaked early and decreased over the course of breathing load before increasing again after breathing load termination (Fig. 3). RBN activation in the amygdala immediately declined and remained low until a steep increase after breathing load termination.

Exploratory associations with clinical variables

RBN who reported higher worst past self-induced vomiting frequencies showed greater BOLD responses during breathing load anticipation in the right amygdala (Figure S3), whereas those with longer durations of remission from $\mathrm{BN}$ showed less activation during breathing load anticipation in the left caudate, right ACC, left PCC, and bilateral amygdala (Figure S4; Table S5). These clusters associated with BN-specific clinical variables did not overlap with task-related clusters shown in Table 1, and there were no significant associations of these clinical variables with BOLD response during breathing load or post-breathing load conditions.

In both RBN and CW, somatic VAS ratings were associated with striatal, ACC, and mid-cingulate activation during the anticipation condition (Table S6). In the RBN group, lateral PFC and putamen clusters in which anticipatory activation was inversely associated with pleasantness ratings overlapped with clusters in which RBN showed hyperactivation relative to $\mathrm{CW}$. There were no significant associations of current depressive or anxiety symptoms, years of education, or CPT performance with BOLD response in either group, suggesting these variables likely did not contribute to group differences in mean peak activation. Results of additional sensitivity analyses indicated that there were no significant differences in activation between participants with and without past $\mathrm{AN}$, and lowest past BMI was not significantly associated with activation in the RBN group during breathing load anticipation. Group differences in mean peak activation remained statistically significant in repeated analyses excluding RBN women with past major depression, a past alcohol use disorder, and those with a past substance use disorder.

\section{DISCUSSION}

This study is the first to test whether BN is associated with aberrant neural processing of non-painful aversive interoceptive events. Compared with CW, RBN showed increased activation in mid-insula, striatum, lateral PFC, amygdala, dACC, and PCC during anticipation of an unpleasant breathing load. Groups did not differ in mean peak activation during other task conditions. However, time-course analyses revealed that RBN showed early initial peaks in activation followed by a decline in activation during the aversive experience, whereas CW showed a steady increase in activation during the sustained aversive breathing load. Exploratory regressions in RBN indicated that greater limbic activation during anticipation of an aversive interoceptive state could contribute to or result from compensatory behaviors, and striatal and prefrontal hyperactivation may be linked to the perceived aversiveness of an internal experience. In addition, anticipatory limbic, striatal, and cingulate activation may decrease with longer bulimic symptom abstinence. Taken together, these findings suggest excessive activation before and an abnormal decline in activation during aversive interoceptive events in RBN. These alterations may make it particularly difficult to integrate expectations about homeostatic state changes with experiences of those changes, ultimately promoting binge eating and purgingbehaviors that result in homeostatic extremes.

Contrary to our hypotheses, during breathing load anticipation, the RBN group showed hyperactivation in regions involved in aversive prediction errors and the expectation and recall of aversive experience [14, 36, 53-55]. The directionality of these findings contrasts with prior findings of decreased anticipatory and prediction error signaling for taste rewards among individuals ill with BN $[24,56]$. These differences could be related to prior studies' use of appetitive instead of aversive stimuli and the distinct neural circuitry implicated in appetitive and aversive processing [57]. For example, prior findings of reduced prediction error signaling for sucrose were all in regions implicated primarily in appetitive processing or both appetitive and aversive processing (bilateral anterior insula, right putamen, right amygdala, and left orbitofrontal cortex) [56-59], whereas our findings of increased activation during aversive anticipation were all in regions implicated primarily in aversive processing or both appetitive and aversive processing (bilateral mid-insula, bilateral putamen, right amygdala, bilateral dACC, and PCC) [57-59]. 


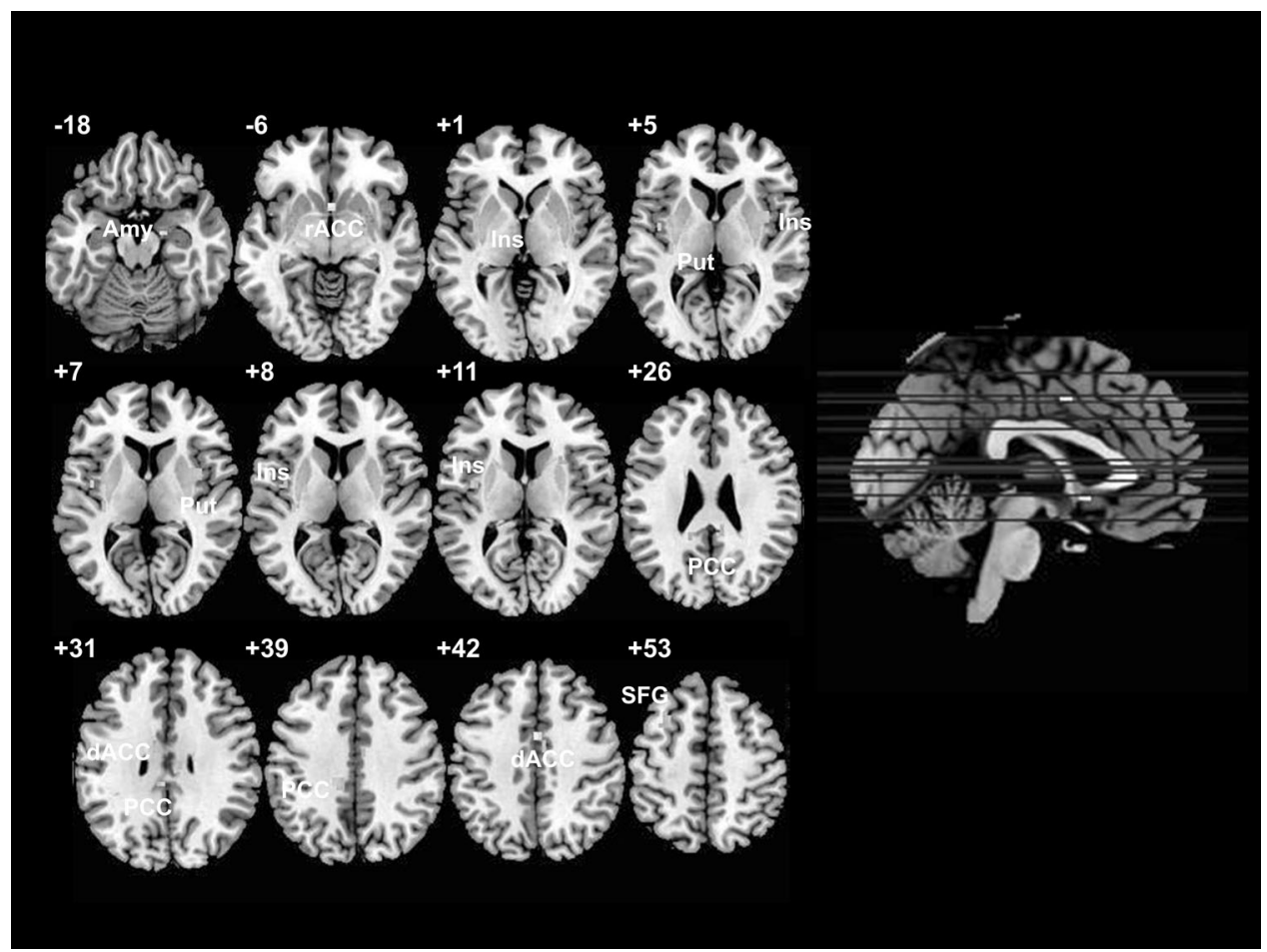

Fig. 2 Increased activation during breathing load anticipation in women remitted from bulimia nervosa relative to controls. Corresponding statistics are presented in Table 1. No statistically significant between-group differences were found during or after the breathing load. Amy amygdala, $r A C C$ rostral anterior cingulate cortex, Ins insula, Put putamen, $P C C$ posterior cingulate cortex, $d A C C$ dorsal anterior cingulate cortex, SFG superior frontal gyrus

Table 2. Group $\times$ Time interactions: mixed effects time series analysis

\begin{tabular}{|c|c|c|c|c|c|c|c|}
\hline \multirow[b]{2}{*}{ Hem } & \multirow[b]{2}{*}{ Region } & \multirow[b]{2}{*}{ BA } & \multirow[b]{2}{*}{ voxels } & \multicolumn{3}{|c|}{$\begin{array}{l}\text { Center of mass } \\
\text { MNI coordinates }\end{array}$} & \multirow[b]{2}{*}{$F$} \\
\hline & & & & $\mathrm{x}$ & $y$ & z & \\
\hline $\mathrm{L}$ & Posterior insula & 13 & 14 & -38 & -19 & 3 & $2.56^{\mathrm{a}}$ \\
\hline L & Rostral anterior cingulate & 32 & 3 & -1 & 21 & -7 & 2.12 \\
\hline $\mathrm{L}$ & Rostral anterior cingulate & 32 & 3 & -9 & 39 & 3 & 2.10 \\
\hline $\mathrm{R}$ & Dorsal anterior cingulate & 24 & 3 & 3 & 6 & 36 & 2.40 \\
\hline $\mathrm{R}$ & Middle frontal gyrus & 6 & 17 & 29 & 4 & 54 & $2.31^{\mathrm{a}}$ \\
\hline $\mathrm{R}$ & Middle frontal gyrus & 45 & 12 & 45 & 20 & 21 & $2.42^{\mathrm{a}}$ \\
\hline R & Middle frontal gyrus & 9 & 9 & 32 & 29 & 34 & $2.21^{\mathrm{a}}$ \\
\hline $\mathrm{R}$ & Middle frontal gyrus & 9 & 5 & 46 & 13 & 29 & 2.13 \\
\hline $\mathrm{L}$ & Middle frontal gyrus & 9 & 4 & -36 & 30 & 28 & 2.13 \\
\hline $\mathrm{L}$ & Posterior cingulate & 23 & 22 & 0 & -34 & 26 & $2.30^{\mathrm{a}}$ \\
\hline $\mathrm{R}$ & Posterior cingulate & 24 & 3 & 14 & -19 & 36 & 2.11 \\
\hline $\mathrm{R}$ & Amygdala & & 4 & 27 & 1 & -27 & $2.88^{\mathrm{a}}$ \\
\hline
\end{tabular}

All results presented were small-volume corrected with Monte Carlo simulations (via Analysis of Functional Neurolmages 3dClustSim with ACF correction) to guard against false-positive results (per voxel $p<0.001$, cluster-wise $p<0.05$ ). Coordinates are presented in LPI format

Hem hemisphere, $L$ left, $R$ right, $B A$ Brodmann area

${ }^{a}$ Clusters that are statistically significant after more stringent small-volume correction (per voxel $p<0.001$, cluster-wise $p<0.01$ )

The directionality of our findings may also differ from those of previous BN studies because in prior paradigms, anticipatory stimuli were strongly predictive of subsequent experience, and predictive probabilities were learned rather than explicitly stated to participants. The predictive visual cue in the breathing load task signaled only a $25 \%$ chance of upcoming load. Therefore, increased anticipatory activation in RBN may indicate an exaggerated neural signal for both uncertainty and anticipation of an aversive experience.

Despite differences from appetitive studies in $\mathrm{BN}$, our results are consistent with increased anticipatory neural responses for aversive stimuli in depressed and anxious populations [60,61], increased striatal and limbic activation during breathing load anticipation in adults low in resilience [62], and increased insula and cingulate activation during hyperventilation anticipation among individuals with elevated fear of somatic symptoms [63]. Our sensitivity analyses suggest that this increased anticipatory response in RBN was not better accounted for by elevated anxiety or depressive symptoms. However, to our knowledge, no one has directly compared BN to mood and anxiety disorders to test whether increased limbic activation during anticipation of an aversive internal experience represents a transdiagnostic trait.

Our findings of altered anticipatory activation in mid-insula, specifically, are consistent with data suggesting an important role for mid-insula activation in the anticipation [64] and recall [55] of aversive interoceptive stimuli in health and depression. The midinsula is hypothesized to relay interoceptive information to limbic and striatal structures, thereby promoting adaptive behaviors to maintain homeostasis $[12,65]$. Exaggerated anticipatory activation in mid-insula, as well as limbic and striatal regions, may interfere with such allostatic signaling in BN. Results of our exploratory analyses preliminarily suggest that increased anticipatory limbic activation is associated with more frequent past self-induced vomiting frequency but could represent a state marker of the illness that attenuates with prolonged symptom abstinence. Of note, increased anticipatory mid-insula activation in RBN directly contrasts with decreased anticipatory mid-insula activation in RAN [33]. Whether opposite responses during the anticipation of aversive body state change drive the predominant symptoms 


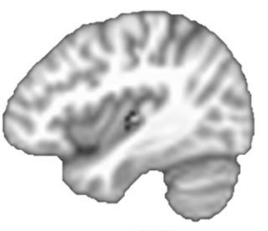

$x=-38$

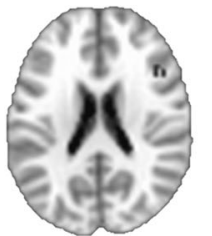

$z=+21$

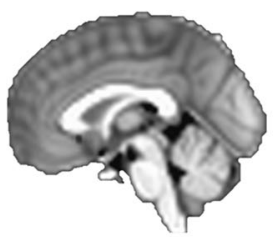

$x=0$

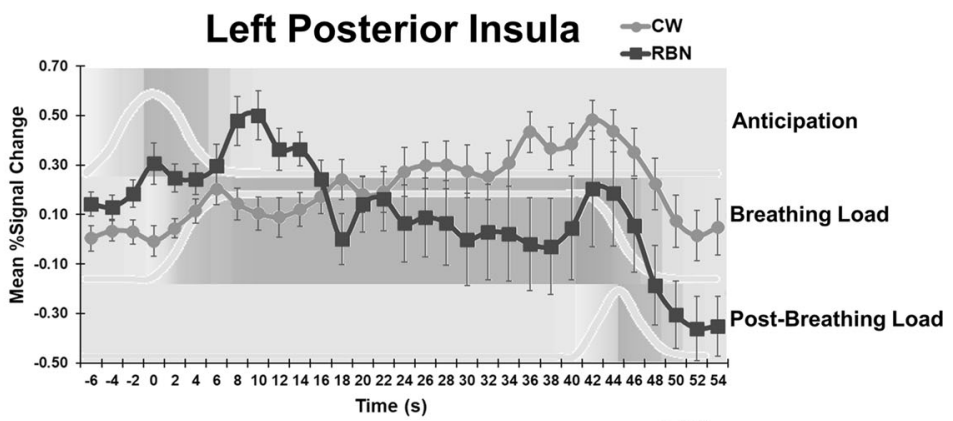

Right MFG

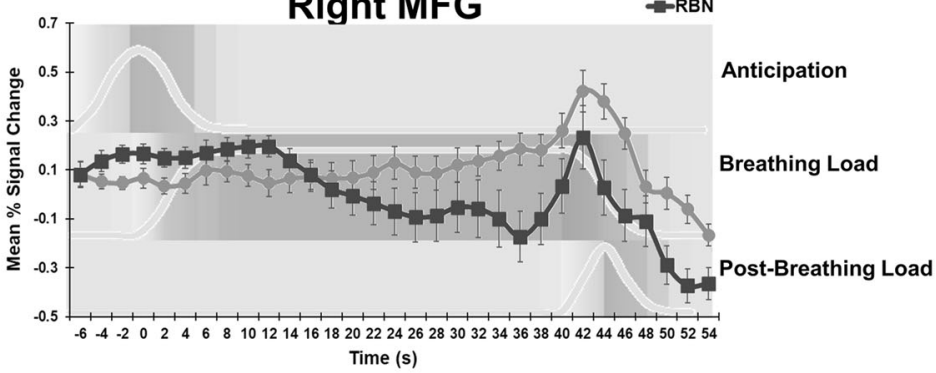

Time (s)

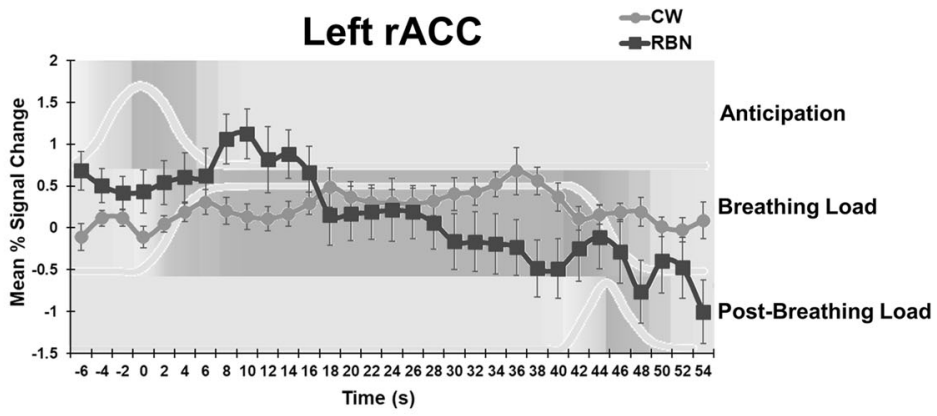

Left PCC $\Rightarrow$-RBN
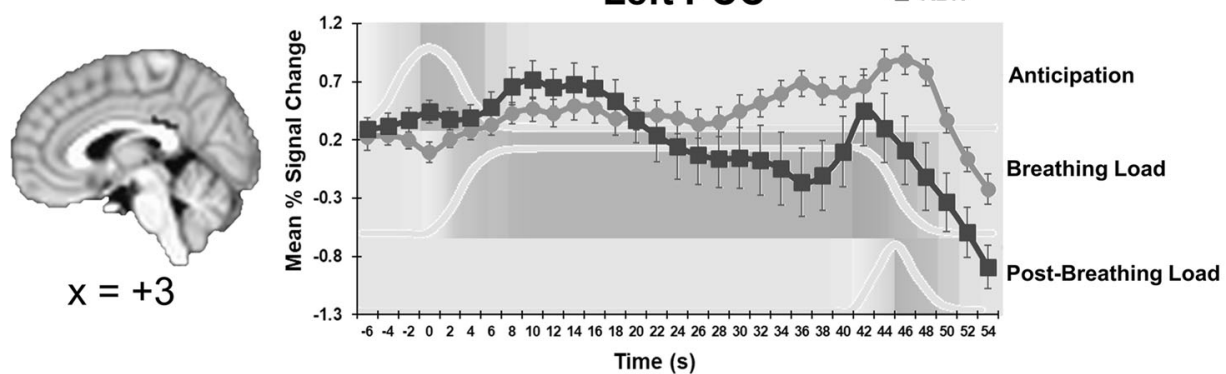

Right Amygdala $\underset{\rightarrow \mathrm{RBN}}{\mathrm{CW}}$

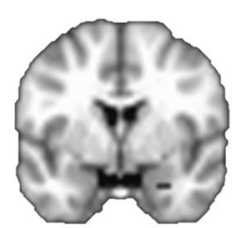

$y=0$

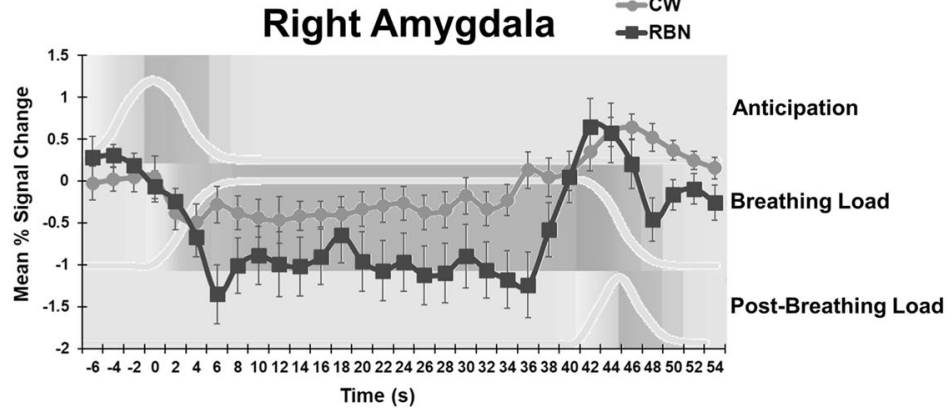

Fig. 3 Group differences in activation time course. Group $x$ Time interaction results suggest that the time course of activation in RBN statistically significantly differed from that of CW. Time-course graphs for anticipation, breathing load, and post-breathing load conditions are shown in in light gray and with gray shading the background. Error bars represent standard error of the mean. Additional clusters in which time course differed between groups are shown in Table 2. RBN women remitted from bulimia nervosa, CW healthy control women, MFG middle frontal gyrus, $r A C C$ rostral anterior cingulate cortex, PCC posterior cingulate cortex 
characteristic of these disorders (binge eating and purging versus extreme dietary restriction) remains to be explored.

Time-course analyses across conditions revealed group differences undetected by our primary analyses, which averaged activation within conditions. The BOLD signal in RBN showed an aberrant pattern of an initial early spike followed by a decline over the course of the breathing load. This BOLD time course in RBN is opposite to that seen in RAN-an initial dip followed by a steeply rising climb in activation over the course of breathing load [33]. These opposite time-course patterns, particularly in the cingulate and right MFG, which, via projections to the anterior insula, likely regulate cognitive reactions during interoceptive experience [15-17], may contribute to or reflect the excessive cognitive control characteristic of $\mathrm{AN}$ and deficient cognitive control characteristic of BN [66]. Consistent with this notion, women ill with $\mathrm{BN}$ relative to $\mathrm{CW}$ show an abnormally abrupt decline in cingulate activation during the engagement of inhibitory control on the Simon Spatial Incompatibility Task [67]. Alternatively, declining activation in the lateral PFC and posterior insula, regions involved in processing stressful events, may reflect adaptive habituation to an aversive internal experience [68]. Such exaggerated habituation could have contributed to recovery in our RBN sample. More frequent assessments of perceived intensity and aversiveness of the experience are needed to test this possibility.

RBN participants did not significantly differ from CW on postscan ratings of somatic sensations or pleasantness, unpleasantness, or intensity of the breathing load experience. However, lateral PFC and putamen clusters inversely associated with pleasantness ratings in RBN overlapped with clusters showing task-related group differences, suggesting that these ratings may be linked to anticipatory hyperactivation in these regions. Future studies incorporating neuroimaging with measures of interoceptive accuracy (e.g., [69]), sensibility (beliefs about interoception), and awareness (correspondence between confidence and accuracy) will be important to fully characterize the conscious and unconscious interoceptive alterations that may promote BN.

\section{LIMITATIONS}

Several limitations should be noted. Our sample size was modest and included only RBN, and physiological and self-report data were missing from some participants. Moreover, we did not assess carbon dioxide levels. Prior studies using the breathing load task have not detected group differences in carbon dioxide levels, but future research would benefit from examining associations of breathing rate, expired carbon dioxide, and neural activation in $\mathrm{BN}$. In addition, the CPT in the current paradigm requires attentional control, and our design does not permit comparison of trials with and without this button-pressing task to isolate activation related to the added attentional demand. Additional studies should be conducted without this CPT. Finally, despite our statistical investigation of the effects of past comorbidities, future studies with larger sample sizes are necessary to comprehensively test the potential impact of comorbid symptoms and disorders on altered interoception in BN.

\section{CONCLUSION AND FUTURE DIRECTIONS}

The current study is the first to measure the neural correlates of interoceptive perturbation in $\mathrm{BN}$, and our findings add to mounting evidence suggesting that anticipation and experience of body sensations is altered in eating disorders [24, 33, 56, 70-72]. Our results also highlight a potential role for aberrant temporal characteristics of interoceptive processing in BN. These findings may have important implications for understanding BN maintenance and enhancing interventions. Interoceptive signals inform motivated approach and avoidance behaviors, and in anxiety and mood disorders, avoidant symptoms have been conceptualized as attempts to compensate for the pronounced difference between signals for expectation and experience of aversive body states [73]. Similarly, in BN, neural hyperactivation during the anticipation of an aversive interoceptive experience coupled with abnormal temporal decline of the BOLD response during aversive experience may contribute to the dynamic interplay of avoidant and arousal-seeking behaviors characteristic of the disorder [74]. Pharmacological or neuromodulatory interventions could be targeted to stabilize these interoceptive responses. In addition, as symptoms persist in as many as $70 \%$ of patients who receive first-line psychotherapies for the disorder [75], psychosocial interventions may benefit from an increased focus on anticipatory cognitions and behaviors.

Further investigation of interoceptive dysregulation in $\mathrm{BN}$ is warranted. Since our anticipatory cues were only $25 \%$ predictive of subsequent breathing load, breathing loads were partially unexpected. Abnormally declining activation in RBN during this sustained, partially unexpected aversive stimulus may be consistent with reduced activation observed in symptomatic BN in response to unexpected, brief tastes of sucrose [56]. As theoretical models have posited for a range of psychopathology [12], BN may be characterized by aversive interoceptive prediction errors, and perhaps specifically altered temporal dynamics of these prediction errors. Applications of computational modeling to tasks that include expected, unexpected, and omitted interoceptive perturbations are needed to formally test this hypothesis. Replication of our methods in larger, currently symptomatic samples is also needed to determine whether interoceptive alterations are traitlike and how they may relate directly to dysregulated eating behavior. Additionally, since our sample included women with and without past $A N$, and results were opposite to those in RAN with no history of BN [33], longitudinal research should investigate whether the observed neural responses represent biomarkers for eventual BN development. Future research in BN should explicitly examine the rewarding and aversive aspects of the internal state changes associated with bulimic symptoms and how these may relate to neural processing of interoceptive stimuli more broadly.

\section{FUNDING AND DISCLOSURE}

The authors declare no conflict of interest. This research and preparation of this manuscript were supported in part by grants from the National Institute of Mental Health to WHK (R01 MH042984), WHK and UFB (R01 MH092793), and LAB (F32 $\mathrm{MH} 108311)$, the Price Foundation, and the Hilda and Preston Davis Foundation. The contents of this manuscript are solely the responsibility of the authors and do not necessarily represent the official view of the NIH. Portions of the data herein were presented at annual meetings of the Society of Biological Psychiatry and the American College of Neuropsychopharmacology.

\section{ACKNOWLEDGEMENTS}

The authors thank Miki Ogasawara for assistance with fMRI data collection and preprocessing. We also thank Daria Orlowska, Grace Rasmusson, Laura Greathouse, Raesana Williams, and Jocelyn Danielle Bulante for assistance with participant screening and data collection. Finally, we thank the women who participated in this study for their time.

\section{ADDITIONAL INFORMATION}

Supplementary Information accompanies this paper at (https://doi.org/10.1038/ s41386-019-0361-4).

Publisher's note: Springer Nature remains neutral with regard to jurisdictional claims in published maps and institutional affiliations. 


\section{REFERENCES}

1. American Psychiatric Association. Diagnostic and Statistical Manual of Mental Disorders: Fifth Edition (DSM-5). Washington. DC: American Psychiatric Association; 2013.

2. Santangelo P, Reinhard I, Mussgay L, Steil R, Sawitzki G, Klein C, et al. Specificity of affective instability in patients with borderline personality disorder compared to posttraumatic stress disorder, bulimia nervosa, and healthy controls. J Abnorm Psychol. 2014;123:258-72. https://doi.org/10.1037/a0035619.

3. Craig A. Interoception: the sense of the physiologial condition of the body. Curr Opin Neurobiol. 2003;13:500-5.

4. Craig A. Interoception and emotion: a neuroanatomical perspective, In: Lewis, M., Haviland-Jones, J. M. and Barrett, L. F., editors. Handbook of Emotions, The Guildford Press; New York, 2008.

5. Klabunde $M$, Collado D, Bohon C. An interoceptive model of bulimia nervosa: a neurobiological systematic review. J Psychiatr Res. 2017;94:36-46. https://doi.org/ 10.1016/j.jpsychires.2017.06.009.

6. Lautenbacher S, Pauls AM, Strian F, Pirke K-M, Krieg J-C. Pain sensitivity in anorexia nervosa and bulimia nervosa. Biol Psychiatry. 1991;29:1073-8. https:// doi.org/10.1016/0006-3223(91)90249-L.

7. Stein D, Kaye W, Matsunaga H, Myers D, Orbach I, Har-Even D, et al. Pain perception in recoverd bulimia nervosa patients. Int J Eat Disord. 2003;34:331-6.

8. Walsh BT, Zimmerli E, Devlin MJ, Guss J, Kissileff HR. A disturbance of gastric function in bulimia nervosa. Biol Psychiatry. 2003;54:929-33.

9. Klabunde M, Acheson D, Boutelle K, Matthews S, Kaye W. Interoceptive sensitivity deficits in women recovered from bulimia nervosa. Eat Behav. 2013;14:488-92.

10. Pollatos $\mathrm{O}$, Georgiou E. Normal interoceptive accuracy in women with bulimia nervosa. Psychiatry Res. 2016;240:328-32. https://doi.org/10.1016/j.psychres.2016.04.072.

11. Khalsa SS, Adolphs R, Cameron OG, Critchley HD, Davenport PW, Feinstein JS, et al. Interoception and mental health: a roadmap. Biol Psychiatry Cogn Neurosci Neuroimaging. 2018;3:501-13. https://doi.org/10.1016/j.bpsc.2017.12.004.

12. Barrett LF, Simmons WK. Interoceptive predictions in the brain. Nat Rev Neurosci. 2015;16:419-29. https://doi.org/10.1038/nrn3950.

13. Menon M, Jensen J, Vitcu I, Graff-Guerrero A, Crawley A, Smith M, et al. Temporal difference modeling of the blood-oxygen level dependent response during aversive conditioning in humans: effects of dopaminergic modulation. Biol Psychiatry. 2007;62:765-72.

14. Delgado $M$, Li J, Schiller D, Phelps E. The role of the striatum in aversive learning and aversive prediction errors. Philos Trans R Soc B. 2008;363:3787-800.

15. Simmons WK, Avery JA, Barcalow JC, Bodurka J, Drevets WC, Bellgowan P. Keeping the body in mind: insula functional organization and functional connectivity integrate interoceptive, exteroceptive, and emotional awareness. Human Brain Mapp. 2013;34:2944-58. https://doi.org/10.1002/hbm.22113.

16. Hayes DJ, Northoff G. Common brain activations for painful and non-painful aversive stimuli. BMC Neurosci. 2012;13:60. https://doi.org/10.1186/1471-2202-13-60.

17. Critchley HD, Wiens S, Rotshtein P, Ohman A, Dolan RJ. Neural systems supporting interoceptive awareness. Nat Neurosci. 2004;7:189-95.

18. Peiffer C. Dyspnea relief: more than just the perception of a decrease in dyspnea. Respir Physiol Neurobiol. 2009;167:61-71.

19. Frank G, Shott M, Hagman J, Mittal V. Alterations in brain structures related to taste reward circuitry in ill and recovered anorexia nervosa and in bulimia nervosa. Am J Psychiatry. 2013;170:1152-60. https://doi.org/10.1176/appi. ajp.2013.12101294.

20. Frank GKW, Shott ME, Riederer J, Pryor TL. Altered structural and effective connectivity in anorexia and bulimia nervosa in circuits that regulate energy and reward homeostasis. Transl Psychiatry. 2016;6:e932. https://doi.org/10.1038/ tp.2016.199.

21. Frank G, Wagner A, Brooks-Achenbach S, McConaha C, Skovira K, Aizenstein H, et al. Altered brain activity in women recovered from bulimic type eating disorders after a glucose challenge. A pilot study. Int J Eat Disord. 2006;39: 76-9.

22. Wagner A, Simmons AN, Oberndorfer TA, Frank GKW, McCurdy-McKinnon D, Fudge $\mathrm{J}$, et al. Altered sensitization patterns to sweet food stimuli in patients recovered from anorexia and bulimia nervosa. Psychiatry Res: Neuroimaging. 2015;234:305-13. https://doi.org/10.1016/j.pscychresns.2015.10.010.

23. Monteleone AM, Monteleone P, Esposito F, Prinster A, Volpe U, Cantone E, et al. Altered processing of rewarding and aversive basic taste stimuli in symptomatic women with anorexia nervosa and bulimia nervosa: An fMRI study. J Psychiatr Res. 2017;90:94-101. https://doi.org/10.1016/j.jpsychires.2017.02.013.

24. Bohon C, Stice E. Reward abnormalities among women with full and subthreshold bulimia nervosa: a functional magnetic resonance imaging study. Int J Eat Disord. 2011;44:585-95. https://doi.org/10.1002/eat.20869.

25. Oberndorfer T, Frank G, Fudge J, Simmons A, Paulus M, Wagner A, et al. Altered insula response to sweet taste processing after recovery from anorexia and bulimia nervosa. Am J Psychiatry. 2013;214:132-41. https://doi.org/10.1176/appi. ajp.2013.11111745.
26. Ceunen E, Vlaeyen JWS, Van Diest I. On the origin of interoception. Front Psychol. 2016;7:743. https://doi.org/10.3389/fpsyg.2016.00743.

27. Heatherton T, Baumeister T. Binge eating as an escape from self-awareness. Psychol Bull. 1991;110:86-108.

28. Hayaki J. Negative reinforcement eating expectancies, emotion dysregulation, and symptoms of bulimia nervosa. Int J Eat Disord. 2009;42:552-6. https://doi. org/10.1002/eat.20646.

29. Culbert KM, Racine SE, Klump KL. Research review: what we have learned about the causes of eating disorders-a synthesis of sociocultural, psychological, and biological research. J Child Psychol Psychiatry. 2015;56:1141-64.

30. Penas-Lledo E, Vaz FJ, Ramos MI, Waller G. Impulsive behaviors in bulimic patients: relation to general psychopathology. Int J Eat Disord. 2002;32:98-102.

31. Stewart JL, Juavinett AL, May AC, Davenport PW, Paulus MP. Do you feel alright? Attenuated neural processing of aversive interoceptive stimuli in current stimulant users. Psychophysiology. 2015;52:249-62.

32. Stewart JL, May AC, Poppa T, Davenport PW, Tapert SF, Paulus MP. You are the danger: attenuated insula response in methamphetamine users during aversive interoceptive decision-making. Drug Alcohol Depend. 2014;142:110-9. https:// doi.org/10.1016/j.drugalcdep.2014.06.003.

33. Berner LA, Simmons AN, Wierenga CE, Bischoff-Grethe A, Paulus MP, Bailer UF, et al. Altered interoceptive activation before, during, and after aversive breathing load in women remitted from anorexia nervosa. Psychol Med. 2018;48:142-54. https://doi.org/10.1017/s0033291717001635.

34. Kaye W, Wierenga C, Bailer U, Simmons A, Wagner A, Bischoff-Grethe A. Does a shared neurobiology for foods and drugs of abuse contribute to extremes of food ingestion in anorexia and bulimia nervosa? Biol Psychiatry. 2013;73:836-42. https://doi.org/10.1016/j.biopsych.2013.01.002.

35. Dawe S, Loxton N. The role of impulsivity in the development of substance use and eating disorders. Neurosci Biobehav Rev. 2004;29:343-51.

36. Moscarello JM, LeDoux JE. The contribution of the amygdala to aversive and appetitive pavlovian processes. Emot Rev. 2013;5:248-53. https://doi.org/ 10.1177/1754073913477508.

37. American Psychiatric Association. Diagnostic and Statistical Manual of Mental Disorders: Fouth Edition (DSM-IV). Washington, DC: American Psychiatric Association; 1994.

38. Haase L, May AC, Falahpour M, Isakovic S, Simmons AN, Hickman SD, et al. A pilot study investigating changes in neural processing after mindfulness training in elite athletes. Front Behav Neurosci. 2015;9:229. https://doi.org/10.3389/ fnbeh.2015.00229.

39. Paulus MP, Flagan T, Simmons AN, Gillis K, Kotturi S, Thom N, et al. Subjecting elite athletes to inspiratory breathing load reveals behavioral and neural signatures of optimal performers in extreme environments. PloS ONE. 2012;7: e29394.

40. Galli G, Shukla A, Simmons AN, Davenport PW, Paulus MP. Sex differences in the neural processing of aversive interoceptive events: the benefit of relief. PLoS ONE. 2013;8:e84044. https://doi.org/10.1371/journal.pone.0084044.

41. Berk L, Stewart JL, May AC, Wiers RW, Davenport PW, Paulus MP, et al. Under pressure: adolescent substance users show exaggerated neural processing of aversive interoceptive stimuli. Addiction. 2015;110:2025-36. https://doi.org/ 10.1111/add.13090.

42. Haase L, Thom NJ, Shukla A, Davenport PW, Simmons AN, Paulus MP, et al. Mindfulness-based training attenuates insula response to an aversive interoceptive challenge. Soc Cogn Affect Neurosci. 2014;11:nsu042.

43. Garner DM. Eating Disorder Inventory-2 Professional Manual. Odessa: Psychological Assessment Resources; 1991.

44. Beck AT, Steer RA, Brown GK. Manual for the Beck Depression Inventory-II. San Antonio: Psychological Corporation; 1996.

45. Cloninger CR, Przybeck TR, Svrakic DM. The Temperament and Character Inventory (TCI): A guide to its development and use: Center for Psychobiology of Personality. St. Louis: Washington University; 1994.

46. Spielberger CD, Gorsuch RL, Lushene RD. State-Trait Anxiety Inventory Manual. Palo Alto: Consulting Psychologists Press; 1970.

47. Chen G, Saad ZS, Britton JC, Pine DS, Cox RW. Linear mixed-effects modeling approach to FMRI group analysis. Neurolmage. 2013;73:176-90. https://doi.org/ 10.1016/j.neuroimage.2013.01.047.

48. Desikan RS, Ségonne F, Fischl B, Quinn BT, Dickerson BC, Blacker D, et al. An automated labeling system for subdividing the human cerebral cortex on MRI scans into gyral based regions of interest. Neuroimage. 2006;31:968-80. https:// doi.org/10.1016/j.neuroimage.2006.01.021.

49. Bissonette GB, Gentry RN, Padmala S, Pessoa L, Roesch MR. Impact of appetitive and aversive outcomes on brain responses: linking the animal and human literatures. Front Syst Neurosci. 2014;8:24. https://doi.org/10.3389/fnsys.2014.00024.

50. Eklund $A$, Nichols $T$, Knutsson $H$. Cluster failure: Why $f M R I$ inferences for spatial extent have inflated false-positive rates. Proc Natl Acad Sci USA. 2016;113:7900-5. https://doi.org/10.1073/pnas.1602413113. 
51. Huber P. Robust estimation of location parameter. Ann Math Stat. 1964;35:73-101.

52. Nichols T, Brett M, Anderson J, Wager T, Poline J. Valid conjunction inference with the minimum statistic. Neuroimage. 2005;25:653-60.

53. Simmons A, Matthews SC, Stein MB, Paulus MP. Anticipation of emotionally aversive visual stimuli activates right insula. Neuroreport. 2004;15: 2261-5.

54. Carlson JM, Greenberg T, Rubin D, Mujica-Parodi LR. Feeling anxious: anticipatory amygdalo-insular response predicts the feeling of anxious anticipation. Soc Cogn Affect Neurosci. 2011;6:74-81. https://doi.org/10.1093/scan/nsq017.

55. DeVille DC, Kerr KL, Avery JA, Burrows K, Bodurka J, Feinstein J, et al. The neural bases of interoceptive encoding and recall in healthy adults and adults with depression. Biol Psychiatry Cogn Neurosci Neuroimaging. 2018;3:546-54. https:// doi.org/10.1016/j.bpsc.2018.03.010.

56. Frank GKW, Reynolds JR, Shott ME, O'Reilly RC. Altered temporal difference learning in bulimia nervosa. Biol Psychiatry. 2011;70:728-35.

57. Hayes D, Ducana N, Xua J, Northoff G. A comparison of neural responses to appetitive and aversive stimuli in humans and other mammals. Neuro Biobehav Rev. 2014;45:350-68. https://doi.org/10.1016/j.neubiorev.2014.06.018.

58. Craig AD. Forebrain emotional asymmetry: a neuroanatomical basis? Trends Cogn Sci. 2005;9:566-71. https://doi.org/10.1016/j.tics.2005.10.005.

59. Strigo IA, Craig AD. Interoception, homeostatic emotions and sympathovagal balance. Philos Trans R Soc B Biol Sci. 2016;371:pii: 20160010.

60. Strigo IA, Simmons AN, Matthews SC, Craig A, Paulus MP. Association of major depressive disorder with altered functional brain response during anticipation and processing of heat pain. Arch Gen Psychiatry. 2008;65:1275-84. https://doi. org/10.1001/archpsyc.65.11.1275.

61. Paulus MP, Stein MB. Interoception in anxiety and depression. Brain Struct Funct. 2010;214:451-63. https://doi.org/10.1007/s00429-010-0258-9.

62. Haase L, Stewart JL, Youssef B, May AC, Isakovic S, Simmons AN, et al. When the brain does not adequately feel the body: links between low resilience and interoception. Biol Psychol. 2016;113:37-45. https://doi.org/10.1016/j. biopsycho.2015.11.004.

63. Holtz K, Pané-Farré CA, Wendt J, Lotze $M$, Hamm AO. Brain activation during anticipation of interoceptive threat. Neurolmage. 2012;61:857-65. https://doi.org/ 10.1016/j.neuroimage.2012.03.019.

64. Yágüez L, Coen S, Gregory LJ, Amaro E, Altman C, Brammer MJ, et al. Brain response to visceral aversive conditioning: a functional magnetic resonance imaging study. Gastroenterology. 2005;128:1819-29. https://doi.org/10.1053/j. gastro.2005.02.068.

65. Kleckner IR, Zhang J, Touroutoglou A, Chanes L, Xia C, Simmons WK, et al. Evidence for a large-scale brain system supporting allostasis and interoception in humans. Nat Human Behav. 2017;1:0069. https://doi.org/10.1038/s41562-0170069.

66. Wierenga C, Ely A, Bischoff-Grethe A, Bailer U, Simmons A, Kaye W. Are extremes of consumption in eating disorders related to an altered balance between reward and inhibition? Front Behav Neurosci. 2014;9:410. https://doi.org/10.3389/ fnbeh.2014.00410.

67. Marsh R, Steinglass JE, Gerber AJ, O'Leary KG, Walsh BT, Peterson BS. Deficient activity in the neural systems that mediate self-regulatory control in bulimia nervosa. Arch General Psychiatry. 2009;66:1-13.

68. Sinha R, Lacadie CM, Constable RT, Seo D. Dynamic neural activity during stress signals resilient coping. Proc Natl Acad Sci USA. 2016;113:8837-42. https://doi. org/10.1073/pnas.1600965113.

69. Garfinkel SN, Manassei MF, Hamilton-Fletcher G, In den Bosch Y, Critchley HD, Engels M. Interoceptive dimensions across cardiac and respiratory axes. Philos Trans R Soc B Biol Sci. 2016;371:pii: 20160014.

70. Strigo I, Matthews S, Simmons A, Oberndorfer T, Reinhardt L, Klabunde M, et al. Altered insula activation during pain anticipation in individuals recovered from anorexia nervosa: evidence of interocetive dysregulation. Int J Eat Disord. 2013;46:23-33. https://doi.org/10.1002/eat.22045.

71. Khalsa S, Craske M, Li W, Vangala S, Strober M, Feusner J. Altered interoceptive awareness in anorexia nervosa: effects of meal anticipation, consumption and bodily arousal. Int J Eat Disord. 2015;48:889-97. https://doi.org/10.1002/ eat.22387.

72. Pearson CM, Chester DS, Powell D, Wonderlich SA, Smith GT. Investigating the reinforcing value of binge anticipation. Int J Eat Disord. 2016;49:539-41. https:// doi.org/10.1002/eat.22547.

73. Paulus MP, Stein MB. An insular view of anxiety. Biol Psychiatry. 2006;60:383-7. https://doi.org/10.1016/j.biopsych.2006.03.042.

74. Pearson CM, Wonderlich SA, Smith GT. A risk and maintenance model for bulimia nervosa: from impulsive action to compulsive behavior. Psychol Rev. 2015;122:516-35. https://doi.org/10.1037/a0039268.

75. Linardon J, Wade TD. How many individuals achieve symptom abstinence following psychological treatments for bulimia nervosa? A meta-analytic review. Int J Eat Disord. 2018;51:287-94. https://doi.org/10.1002/eat.22838. 